Revista Brasileira de

Engenharia Agrícola e Ambiental

v. 15 , n.4, p.331-337, 2011

Campina Grande, PB, UAEA/UFCG - http://www.agriambi.com.br

agriambi Protocolo 097.10 - 26/05/2010 • Aprovado em 24/01/2011

\title{
Potencial erosivo e características das chuvas de Encruzilhada do Sul, RS
}

\author{
Flávio L. F. Eltz ${ }^{1}$, Elemar A. Cassol ${ }^{2} \&$ Pedro B. Pascotini ${ }^{3}$
}

\section{RESUMO}

O objetivo deste trabalho foi determinar a erosividade e algumas características das chuvas de Encruzilhada do Sul, com base no período de 1958 a 1988. Para cada chuva considerada erosiva e com base nos pluviogramas diários, foram digitados a hora e o volume acumulado, sendo os dados processados pelo programa computacional CHUVEROS, que calcula o índice $\mathrm{EI}_{30}$, os totais mensais e anuais, determinando também os padrões hidrológicos das chuvas. 0 coeficiente de chuvas, o período de retorno e a probabilidade de ocorrência, também foram calculados. 0 período de janeiro a março concentra $38 \%$ da erosividade anual, com pico maior em fevereiro $\left(808,5 \mathrm{MJ} \mathrm{mm} \mathrm{ha-1} \mathrm{h}^{-1}\right)$, enquanto de julho a setembro concentra o período com menor potencial erosivo (14\%). N a média, os padrões avançado, intermediário e atrasado têm 48,26 e $26 \%$ do número de chuvas erosivas, perfazendo 51,27 e $22 \%$ do volume médio anual e 55, 27 e 18\% da erosividade média anual. Para Encruzilhada do Sul o valor do índice de erosividade anual é de $5534,3 \mathrm{MJ} \mathrm{mm} \mathrm{ha-1} \mathrm{h}^{-1}$ (Fator "R" da USLE), com período de retorno de 2,3 anos e probabilidade de ocorrência de $40,6 \%$. Verificou-se que o potencial erosivo das chuvas $\left(\mathrm{EI}_{30}\right)$ não apresenta correlação significativa com o coeficiente de chuvas.

Palavras-chave: potencial erosivo da chuva, índice $\mathrm{El}_{30}$, energia cinética, fator $\mathrm{R}$

\section{Erosivity potential and characteristics of rainfalls at Encruzihada do Sul, RS}

\begin{abstract}
The objective of this work was to determine the rainfall erosivity and some characteristics of erosive rainfalls of Encruzilhada do Sul (RS, Brazil), based on the period of 1958 to 1988. For each erosive rainfall, based on daily rainfall charts, the hour and amounts were digited, and processed by the software Chuveros, which calculates the $\mathrm{El}_{30}$ index, the monthly and annual totals, besides to determine the hydrol ogical pattern of each rainfall. The rainfall coefficient, the return period and its occurrence probability were also calculated. The January to $\mathrm{M}$ arch period concentrates $38 \%$ of annual erosivity, with highest peak in February $\left(808.5 \mathrm{MJ} \mathrm{mm} \mathrm{ha-1} \mathrm{h}^{-1}\right)$, while July to September concentrates the smallest erosive potential $(14 \%)$. In the average, the advanced, intermediary and delayed patterns present 48,26 and $26 \%$ of the number of erosive rainfalls per year, respectively, correponding to 51,27 and $22 \%$, respectively, of the annual volume of erosive rainfalls and 55,27 and $18 \%$ of the average annual erosivity. For Encruzilhada do Sul, the mean annual rainfall erosivity index is $5534.3 \mathrm{MJ} \mathrm{mm} \mathrm{ha-1} \mathrm{h}^{-1}$ ("R" Factor of USLE), with a return period of 2.3 years, and occurrence probability of $40.6 \%$. It has been verified that the rainfall erosive potential $\left(\mathrm{EI}_{30}\right)$ does not present significant correlation to the rainfall coefficient.
\end{abstract}

Key words: rainfall erosive potential, $\mathrm{El}_{30}$ index, kinetic energy, $\mathrm{R}$ factor

\footnotetext{
${ }^{1}$ Departamento de Solos/U FSM, 97105-900, Santa Maria, RS. Fone: (55) 3220-8916, Fax: (55) 3220-8256. E-mail: felt女@ccr.ufsm.br 2 Departamento de Solos/FA-U FRGS, Av. Bento Gonçalves, 7712, C.P. 15100, CEP 91540-000, Porto Alegre, RS. Fone: (51) 3308-6855, Fax: (51) 3308-6050. E-mail: cassolea@orion.ufrgs.br

${ }^{3}$ Graduando em Agronomia da UFSM. Fone: (55) 3220-8916, Fax: (55) 3220-8256. E-mail: pedropascotini@hotmail.com; bolsista de Iniciação Científica do $\mathrm{CNPq}$
} 


\section{INTRODUÇÃO}

O conhecimento do potencial erosivo das chuvas de uma região é fator importante para o controle da erosão, principal forma de degradação do solo em áreas agrícolas. As perdas de solo por erosão podem ser estimadas com a utilização de modelos de predição, sendo a Equação Universal de Perdas de Solo (Wischmeier \& Smith, 1978) um dos mais usados. O fator $\mathrm{R}$ desta equação representa o potencial erosivo das chuvas de uma região, tomados como base períodos de 20 a 30 anos de dados pluviográficos. Para cada chuva é determinado o índice $\mathrm{EI}_{30}$, que representa o produto da energia cinética total da chuva $\left(\mathrm{E}, \mathrm{MJ} \mathrm{ha}^{-1}\right)$ pela intensidade máxima em 30 min $\left(\mathrm{I}_{30}, \mathrm{~mm} \mathrm{~h}^{-1}\right)$, com base em um período de $30 \mathrm{~min}$ contínuo de chuva. No Rio Grande do Sul, Morais et al. (1988) concluíram que o Índice $\mathrm{EI}_{30}$ é adequado para estimar o potencial erosivo das chuvas, baseados em correlações entre índices de erosividade da chuva e perdas de solo por erosão. Outros pesquisadores (Lombardi Neto, 1977; Albuquerque et al., 1998), também obtiveram, em diferentes regiões edafoclimáticas brasileiras, a mesma resposta.

Uma característica importante da chuva relacionada com a erosão é o padrão hidrológico. As chuvas foram caracterizadas por Horner \& Jens (1942) em padrões hidrológicos denominados avançados, intermediários e atrasados, respectivamente, quando o pico de maior intensidade da chuva ocorre no primeiro terço, no segundo terço e no terceiro terço do período de duração total da chuva. No Rio Grande do Sul os padrões hidrológicos foram caracterizados por Mehl et al. (2001) em Santa Maria, por Peñalva-Bazzano et al. (2007) em Quaraí, por Cassol et al. (2007) em Ijuí, por Cassol et al. (2008) em São Borja e por Mazurana et al. (2009) para Santa Rosa, com o padrão avançado ocorrendo com maior frequência.

O objetivo do presente trabalho foi determinar o potencial erosivo das chuvas pelo índice $\mathrm{EI}_{30}$, com base em um período de 31 anos (1958 a 1988), estimando o valor do fator "R" para utilização na Equação Universal de Perdas de Solo, seus padrões hidrológicos e o período de retorno das chuvas erosivas para a região de Encruzilhada do Sul.

\section{Material e MÉTOdos}

O município de Encruzilhada do Sul se situa entre as coordenadas de latitude $52^{\circ} 31^{\prime} \mathrm{W}$ e longitude $30^{\circ} 32^{\prime} \mathrm{S}$, em uma altitude de $420 \mathrm{~m}$ na região fisiográfica Serra do Sudeste. Os pluviogramas diários foram obtidos na estação agrometeorológica da FEPAGRO, com amplitude de $10 \mathrm{~mm}$, unidades de $0,1 \mathrm{~mm}$ e tempo de registro de $24 \mathrm{~h}$ e unidades de 10 minutos, sendo a leitura realizada com precisão de $5 \mathrm{~min}$. $\mathrm{O}$ índice $\mathrm{EI}_{30}$ foi determinado para cada chuva individual erosiva, em que cada chuva individual é separada de outra por um período mínimo de $6 \mathrm{~h}$ sem chuva ou com precipitação menor que $1,0 \mathrm{~mm}$; chuva erosiva é toda aquela com 10,0 ou mais $\mathrm{mm}$ de chuva ou com 6,0 mm ou mais em 15 min ou menos.

Para a análise das chuvas nos pluviogramas diários, foram separados os segmentos de mesma inclinação que representam períodos de mesma intensidade. Em planilha foram registrados as horas e minutos do dia, notando-se a variação de intensidade e a chuva acumulada até aquele momento; após digitação os dados foram analisados pelo programa CHUVEROS, desenvolvido pelo Prof. Elemar Antonino Cassol, do Departamento de Solos da UFRGS, obtendo-se a erosividade mensal, anual e média das chuvas, pelo índice $\mathrm{EI}_{30}$ (Wischmeier, 1959) no Sistema Internacional de Unidades (Foster et al., 1981).

A soma dos índices $\mathrm{EI}_{30}$ das chuvas erosivas individuais de cada mês fornece a erosividade mensal, em todos os meses da série de anos analisados. Também se obtiveram médias mensais da erosividade da chuva cuja soma fornece o $\mathrm{EI}_{30}$ anual e a média é o fator "R" da USLE para aplicação em Encruzilhada do Sul.

Determinaram-se os padrões hidrológicos das chuvas analisadas segundo a classificação proposta por Horner \& Jens (1942), que classificam as chuvas em padrões hidrológicos avançado, intermediário e atrasado, respectivamente, quando a localização do pico de maior intensidade ocorre no início (primeiro terço), meio (segundo terço) e fim (terceiro terço) do período de duração da chuva. Com base no padrão hidrológico de cada chuva calculou-se a percentagem do total das chuvas que representam cada padrão hidrológico, em número, volume e erosividade.

Com os dados da série pluviográfica de Encruzilhada do Sul (1958-1988) foram investigadas relações linear e potencial entre a erosividade das chuvas $\left(\mathrm{EI}_{30}\right.$, e seu coeficiente, conforme proposto por Lombardi Neto (1977) e segundo as seguintes expressões:

$$
\begin{gathered}
\mathrm{EI}_{30}=\mathrm{a}+\mathrm{bRc} \\
\mathrm{EI}_{30}=\mathrm{aRc} c^{\mathrm{b}}
\end{gathered}
$$

em que $\mathrm{EI}_{30}$ é o índice de erosividade das chuvas, $\mathrm{MJ} \mathrm{mm} \mathrm{ha}^{-1}$ $\mathrm{h}^{-1}$, a e b são coeficientes de ajuste e Rc é o coeficiente de chuva, em mm, dado pela seguinte relação: $\mathrm{Rc}=\mathrm{Pm}^{2} \mathrm{~Pa}^{-1}$, em que Pm é a precipitação pluvial média mensal, em mm, e Pa é a precipitação pluvial média anual, em mm.

O período de retorno e a probabilidade de ocorrência da erosividade anual foram determinados conforme Schwab et al. (1981), por meio das seguintes expressões:

$$
\begin{gathered}
\mathrm{T}=(\mathrm{N}+1) / \mathrm{m} \\
\mathrm{Pr}=100 / \mathrm{T}
\end{gathered}
$$

em que T é o período de retorno, em anos, no qual o índice de erosividade é igualado ou superado; $\mathrm{N}$ é o número de anos de registro de dados; m é o número de ordem do índice de erosividade da série considerada, quando a erosividade é colocada em ordem decrescente de magnitude e Pr é a probabilidade de ocorrência do índice de erosividade, em porcentagem.

\section{RESULTADOS E DISCUSSÃO}

Analisaram-se 1118 chuvas erosivas no período 1958-1988 e ocorreram 2233 chuvas não erosivas, o que configura uma 
média anual de 36 chuvas erosivas e 72 não erosivas. Em relação ao número total a porcentagem de chuvas erosivas foi de 33,4 e as chuvas não erosivas de $66,6 \%$; entretanto, em relação ao volume essas chuvas erosivas perfizeram $82,7 \%$, enquanto as chuvas não erosivas perfizeram apenas $17,3 \%$ do volume total de chuvas, neste período de 31 anos (Figura 1).

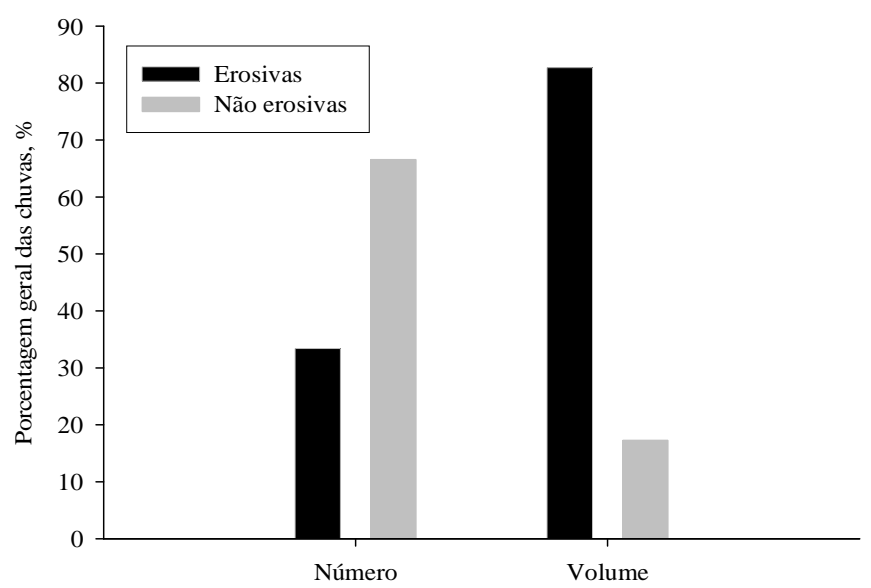

Figura 1. N úmero e volume de chuvas erosivas e não erosivas ocorridas em Encruzilhada do Sul, no período de 1958 a 1988
A precipitação média anual em Encruzilhada do Sul no período de 1958 a 1988, foi de 1279,1 mm (Tabela 1), com o valor mínimo de 662,3 mm ocorrido em 1967 e máximo de 1913,0 mm em 1987. A distribuição mensal da precipitação tem valores médios máximos em junho e agosto, com 124,4 e 119,4 mm, respectivamente, e médio mínimo de $89,9 \mathrm{~mm}$ em abril, com extremos mínimos de $2,3 \mathrm{~mm}$ em maio e $3,8 \mathrm{~mm}$ em dezembro e extremo máximo de 417,2 mm, ocorrido em maio de 1984. A distribuição da precipitação em Encruzilhada do Sul, mostrada na Tabela 1, indica maior frequência de baixas precipitações ao longo do ano e menores frequências de precipitações extremas.

A erosividade mensal de Encruzilhada do Sul (Tabela 2) variou de zero (em vários meses em que não ocorreram chuvas erosivas) até valores altos, como o verificado em fevereiro de 1969, de $4585,1 \mathrm{MJ} \mathrm{mm} \mathrm{ha}^{-1} \mathrm{~h}^{-1}$; este valor representa $74 \%$ da erosividade ocorrida neste ano. No mês de fevereiro de 1969 apenas duas chuvas representaram $71 \%$ desta erosividade, o que as caracteriza como altamente erosivas e reforça a a hipótese de que os referidos eventos extremos são os que, geralmente, causam maiores problemas de erosão. Edwards \& Owens (1991) em Coshocton, Ohio, USA, constataram que cinco eventos de chuva foram responsáveis por $66 \%$ da erosão ocorrida em um período de 28 anos, com mais de 4000 eventos de chuva. O estudo desses eventos é importante em cada município, de forma que possam

Tabela 1. Valores mensais, anuais e médios da precipitação pluvial $(\mathrm{mm})$ em Encruzilhada do Sul, RS, período 19581988 , com os valores extremos em cada mês

\begin{tabular}{|c|c|c|c|c|c|c|c|c|c|c|c|c|c|}
\hline Anos & Jan & Fev & Mar & $A b r$ & Mai & Jun & Jul & Ago & Set & Out & Nov & Dez & Total \\
\hline 1958 & $105,0^{*}$ & $109,5^{*}$ & 70,5 & 145,0 & 103,5 & 178,1 & 105,2 & 198,9 & 209,3 & 129,2 & 123,1 & 106,7 & 1584,0 \\
\hline 1959 & 234,6 & 118,0 & 87,5 & 224,2 & 202,8 & 155,8 & 64,6 & 250,9 & 145,0 & 176,9 & 23,6 & 75,6 & 1759,5 \\
\hline 1960 & $105,0^{*}$ & 88,3 & 133,7 & 79,9 & 51,4 & 154,4 & 214,7 & 182,1 & 182,4 & 197,5 & 72,4 & 62,4 & 1524,2 \\
\hline 1961 & 68,3 & 243,9 & 150,9 & 109,2 & 75,3 & 197,6 & 47,1 & 60,7 & 209,3 & 103,2 & 77,8 & 252,3 & 1595,6 \\
\hline 1962 & 104,4 & 44,1 & 89,4 & 81,2 & 30,8 & 32,4 & 66,1 & 52,2 & 129,0 & 86,9 & 60,1 & 43,0 & 819,6 \\
\hline 1963 & 197,6 & 75,5 & 169,4 & 37,2 & 107,0 & 76,2 & 83,4 & 111,8 & 93,8 & 340,3 & 145,8 & 68,4 & 1506,4 \\
\hline 1964 & 26,5 & 82,6 & 91,2 & 89,6 & 27,8 & 18,3 & 109,2 & 143,1 & 76,4 & 49,1 & 67,8 & 117,6 & 899,2 \\
\hline 1965 & 62,3 & 61,9 & 149,6 & 26,2 & 69,8 & $124,4^{*}$ & 27,1 & 243,2 & 263,7 & 175,5 & 36,1 & 181,1 & 1420,9 \\
\hline 1966 & 73,6 & 161,5 & 168,5 & 83,1 & 2,3 & 147,7 & 60,0 & 63,5 & 18,4 & 60,6 & 33,1 & 68,0 & 940,30 \\
\hline 1967 & 58,6 & 121,5 & 75,3 & 37,2 & 33,5 & 83,8 & 54,6 & 25,7 & 18,9 & 56,7 & 56,6 & 39,9 & 662,3 \\
\hline 1968 & 64,5 & 44,6 & 189,8 & 73,2 & 49,8 & 29,4 & 67,0 & 13,5 & 109,7 & 142,7 & 101,3 & 69,3 & 954,8 \\
\hline 1969 & 81,9 & 323,8 & 53,2 & 30,0 & 49,0 & 85,6 & 51,8 & 20,6 & 19,8 & 56,8 & 161,0 & 46,3 & 979,80 \\
\hline 1970 & 48,0 & 140,0 & 59,5 & 42,6 & 195,0 & 205,8 & 149,7 & 38,6 & 26,2 & 140,0 & 29,6 & 93,0 & 1168,00 \\
\hline 1971 & 63,2 & 126,3 & 34,3 & 19,2 & 69,1 & 220,2 & 47,7 & 41,9 & 89,6 & 90,8 & 34,5 & 93,8 & 930,60 \\
\hline 1972 & 130,6 & 63,7 & 206,7 & 56,6 & 37,1 & 131,5 & 212,1 & 112,3 & 67,6 & $113,5^{*}$ & 33,8 & 80,3 & 1245,8 \\
\hline 1973 & 117,8 & 91,9 & 18,0 & 113,1 & 103,5 & 87,8 & 100,5 & 63,8 & 19,2 & 77,5 & 10,5 & 231,8 & 1035,40 \\
\hline 1974 & 143,9 & 70,0 & 124,7 & 15,0 & 55,1 & 183,4 & 87,1 & 172,2 & 30,9 & 32,9 & 116,6 & 96,1 & 1127,90 \\
\hline 1975 & 77,1 & 95,0 & 75,7 & 30,9 & 58,4 & 63,4 & 52,9 & 209,0 & 148,3 & 83,7 & 156,6 & 30,1 & 1081,10 \\
\hline 1976 & 110,0 & 48,2 & 204,3 & 139,0 & 147,1 & 39,5 & 173,8 & 96,3 & 104,3 & 83,1 & 85,2 & 125,3 & 1356,10 \\
\hline 1977 & 160,5 & 160,0 & 84,6 & 186,3 & 59,2 & 198,2 & 222,9 & 147,3 & 144,6 & 174,4 & 70,1 & 63,9 & 1672,00 \\
\hline 1978 & 106,7 & 88,4 & 48,8 & 20,4 & 35,2 & 113,2 & 154,9 & 91,9 & 77,7 & 103,2 & 139,1 & 109,7 & 1089,20 \\
\hline 1979 & 19,2 & 38,4 & 97,6 & 136,1 & 116,8 & 33,3 & 106,9 & 182,2 & 100,2 & 81,0 & 104,8 & 179,2 & 1195,70 \\
\hline 1980 & 76,0 & 43,4 & 208,2 & 20,1 & 51,0 & 166,2 & 141,1 & 53,3 & 50,7 & 100,6 & 84,4 & 159,1 & 1154,10 \\
\hline 1981 & 140,8 & 138,9 & 9,0 & 59,4 & 106,6 & 149,6 & 78,8 & 45,6 & 153,9 & 18,3 & 95,7 & 108,7 & 1105,30 \\
\hline 1982 & 19,6 & 150,2 & 45,3 & 10,3 & 68,7 & 115,3 & 165,8 & 138,7 & 126,6 & 150,9 & 245,4 & 64,5 & 1301,30 \\
\hline 1983 & 170,0 & 207,8 & 130,0 & 86,2 & 119,9 & 120,8 & 228,8 & 70,5 & 66,8 & 72,8 & 68,4 & 44,1 & 1386,10 \\
\hline 1984 & 239,2 & 129,6 & 48,3 & 175,8 & 417,2 & 239,3 & 125,1 & 92,6 & 69,7 & 135,2 & 95,0 & 43,5 & 1810,50 \\
\hline 1985 & 82,8 & 103,6 & 252,0 & 225,4 & 95,3 & 138,6 & 164,0 & 195,2 & 160,7 & 21,5 & 5,6 & 72,9 & 1517,60 \\
\hline 1986 & 54,1 & 134,7 & 107,8 & 247,1 & 309,7 & 110,1 & 124,3 & 174,6 & 128,8 & 177,2 & 242,0 & 60,4 & 1870,80 \\
\hline 1987 & 177,8 & 75,2 & 195,6 & 98,3 & 174,5 & 122,7 & 234,3 & 365,2 & 111,5 & 146,4 & 129,2 & 82,3 & 1913,00 \\
\hline 1988 & 134,7 & 14,0 & 26,6 & 90,0 & 18,6 & 132,8 & 73,0 & 48,6 & 219,1 & 139,9 & 144,7 & 3,8 & 1045,80 \\
\hline MÁX & 239,2 & 323,8 & 252,0 & 247,1 & 417,2 & 239,3 & 234,3 & 365,2 & 263,7 & 340,3 & 245,4 & 252,3 & 1913,0 \\
\hline MÍN & 19,2 & 14,0 & 9,0 & 10,3 & 2,3 & 18,3 & 27,1 & 13,5 & 18,4 & 18,3 & 5,6 & 3,8 & 662,3 \\
\hline Média & 105,0 & 109,5 & 109,9 & 89,9 & 98,1 & 124,4 & 116,0 & 119,5 & 108,8 & 113,5 & 91,9 & 92,7 & 1279,1 \\
\hline
\end{tabular}

MÁX - valor máximo da série. MíN - valor mínimo da série. * significa que o valor não foi observado devido à inexistência do dado de chuva, tendo-se utilizado o valor médio do referido mês da série levantada 
Tabela 2. Valores mensais do índice de erosividade $\mathrm{El}_{30}\left(\mathrm{MJ} \mathrm{mm} \mathrm{ha-1} \mathrm{h}^{-1}\right)$ em Encruzilhada do Sul, RS, período 1958-1988, com os valores extremos em cada mês

\begin{tabular}{|c|c|c|c|c|c|c|c|c|c|c|c|c|}
\hline Anos & Jan & Fev & Mar & $A b r$ & Mai & Jun & Jul & Ago & Set & Out & Nov & Dez \\
\hline 1958 & $702,8^{*}$ & $808,5^{*}$ & 216,8 & 1774,0 & 442,6 & 772,0 & 213,3 & 345,2 & 523,0 & 350,9 & 261,8 & 274,0 \\
\hline 1959 & 1625,3 & 962,0 & 362,4 & 260,0 & 1181,8 & 230,7 & 147,0 & 706,8 & 323,4 & 444,6 & 51,8 & 113,0 \\
\hline 1960 & $702,8^{*}$ & 668,1 & 338,0 & 74,5 & 133,1 & 452,9 & 641,9 & 547,2 & 440,9 & 1553,9 & 375,1 & 320,5 \\
\hline 1961 & 403,5 & 3502,1 & 603,9 & 451,0 & 224,2 & 982,2 & 13,9 & 126,1 & 470,1 & 59,8 & 143,9 & 1887,2 \\
\hline 1962 & 1262,0 & 69,5 & 232,3 & 426,6 & 342,8 & 31,6 & 40,4 & 30,2 & 454,0 & 403,3 & 370,5 & 235,2 \\
\hline 1963 & 1536,2 & 288,0 & 2486,3 & 117,5 & 772,9 & 118,4 & 129,9 & 163,2 & 109,9 & 1480,8 & 1034,1 & 178,6 \\
\hline 1964 & 39,7 & 282,0 & 960,8 & 298,0 & 6,8 & 0,0 & 185,3 & 243,6 & 104,6 & 53,5 & 270,7 & 490,7 \\
\hline 1965 & 223,3 & 55,2 & 537,9 & 0,0 & 165,6 & $451,9 *$ & 7,2 & 541,2 & 534,4 & 759,1 & 22,5 & 1233,3 \\
\hline 1966 & 222,4 & 1095,8 & 1236,8 & 330,3 & 0,0 & 713,1 & 90,1 & 233,6 & 17,7 & 135,5 & 55,7 & 252,3 \\
\hline 1967 & 809,0 & 1273,0 & 307,3 & 46,3 & 21,3 & 262,3 & 78,7 & 0,0 & 0,0 & 22,3 & 158,3 & 86,5 \\
\hline 1968 & 105,9 & 192,2 & 997,9 & 142,1 & 97,1 & 25,4 & 151,9 & 0,0 & 194,1 & 652,5 & 314,8 & 221,8 \\
\hline 1969 & 248,8 & 4585,1 & 24,4 & 41,5 & 80,0 & 184,4 & 134,2 & 9,9 & 0,0 & 135,1 & 665,1 & 71,8 \\
\hline 1970 & 17,3 & 1451,3 & 55,3 & 76,4 & 1070,6 & 741,8 & 326,8 & 28,7 & 0,0 & 350,7 & 38,1 & 241,7 \\
\hline 1971 & 94,1 & 1199,1 & 23,0 & 11,2 & 514,6 & 1269,1 & 97,8 & 53,0 & 300,9 & 301,2 & 71,4 & 467,1 \\
\hline 1972 & 244,0 & 174,3 & 986,6 & 214,1 & 83,7 & 495,7 & 739,4 & 208,5 & 278,0 & $435,6^{*}$ & 0,0 & 1308,2 \\
\hline 1973 & 500,7 & 407,5 & 17,2 & 492,9 & 311,3 & 148,2 & 289,2 & 297,9 & 0,0 & 216,6 & 0,0 & 2016,3 \\
\hline 1974 & 966,1 & 49,2 & 549,7 & 27,0 & 63,0 & 962,5 & 139,9 & 463,2 & 38,6 & 32,3 & 268,9 & 569,4 \\
\hline 1975 & 870,3 & 689,3 & 161,5 & 34,3 & 73,8 & 145,9 & 110,0 & 483,9 & 428,5 & 400,6 & 1652,0 & 0,0 \\
\hline 1976 & 240,9 & 222,6 & 2330,4 & 739,5 & 315,1 & 91,3 & 628,0 & 77,8 & 86,3 & 246,7 & 359,0 & 383,9 \\
\hline 1977 & 973,7 & 1650,2 & 821,5 & 748,9 & 124,6 & 956,6 & 625,0 & 419,9 & 543,9 & 1802,6 & 183,8 & 72,8 \\
\hline 1978 & 564,1 & 183,1 & 89,5 & 0,0 & 33,1 & 343,2 & 312,5 & 186,8 & 119,9 & 105,1 & 299,4 & 280,6 \\
\hline 1979 & 38,5 & 54,6 & 496,1 & 256,6 & 440,6 & 96,5 & 98,4 & 313,3 & 483,5 & 143,0 & 514,4 & 1194,2 \\
\hline 1980 & 974,6 & 30,9 & 846,8 & 0,0 & 61,5 & 521,1 & 346,4 & 189,8 & 129,1 & 220,2 & 118,2 & 1376,2 \\
\hline 1981 & 727,6 & 1162,0 & 0,0 & 172,7 & 375,8 & 315,0 & 222,9 & 52,5 & 137,4 & 0,0 & 426,0 & 423,2 \\
\hline 1982 & 69,2 & 773,4 & 135,6 & 0,0 & 106,0 & 896,6 & 279,2 & 336,3 & 165,2 & 587,1 & 1893,1 & 301,3 \\
\hline 1983 & 1014,3 & 1094,3 & 885,7 & 114,9 & 201,4 & 380,7 & 372,6 & 113,4 & 100,7 & 236,4 & 141,5 & 226,9 \\
\hline 1984 & 3421,6 & 564,3 & 98,8 & 463,8 & 2457,8 & 576,8 & 533,7 & 245,9 & 93,5 & 731,9 & 125,3 & 143,7 \\
\hline 1985 & 163,9 & 717,8 & 1817,8 & 1936,6 & 232,9 & 739,2 & 368,1 & 254,7 & 277,4 & 13,5 & 0,0 & 203,2 \\
\hline 1986 & 101,2 & 386,4 & 738,9 & 2640,8 & 2244,6 & 155,9 & 474,3 & 918,8 & 242,7 & 384,0 & 1527,5 & 135,8 \\
\hline 1987 & 2077,5 & 473,0 & 863,6 & 228,7 & 597,2 & 392,9 & 598,1 & 1792,2 & 281,7 & 629,5 & 592,3 & 178,5 \\
\hline 1988 & 846,0 & $836,4^{*}$ & 54,0 & 379,8 & 0,0 & 554,9 & 97,5 & 45,0 & 469,6 & 614,5 & 552,5 & 0,0 \\
\hline Máx & 3421,6 & 4585,1 & 2486,3 & 2640,8 & 2457,8 & 1269,1 & 739,4 & 1792,2 & 543,9 & 1802,6 & 1893,1 & 2016,3 \\
\hline Mín & 17,3 & 30,9 & 0,0 & 0,0 & 0,0 & 0,0 & 7,2 & 0,0 & 0,0 & 0,0 & 0,0 & 0,0 \\
\hline
\end{tabular}

ser conhecidas sua distribuição, frequência e potencial erosivo e tomadas medidas práticas de conservação do solo, que possam prevenir seus danos.

A erosividade média mensal foi máxima em fevereiro(Figura 2), com valor de $836 \mathrm{MJ} \mathrm{mm} \mathrm{ha}^{-1} \mathrm{~h}^{-1}$ e mínima em setembro, com valor de $237 \mathrm{MJ} \mathrm{mm} \mathrm{ha}^{-1} \mathrm{~h}^{-1}$. A distribuição mensal mostra um período em julho, agosto e setembro, com menores valores de erosividade da chuva e outro período em janeiro, fevereiro e março, com valores maiores indicando que, neste último período, maiores cuidados devem ser tomados para evitar a erosão do solo, como ter as áreas agrícolas devidamente cobertas por culturas e/ou resíduos de culturas. Locais com potencial erosivo baixo no período de inverno também foram encontrados no Rio Grande do Sul, por Cogo et al. (2006) para Santa Maria, por Peñalva-Bazzano et al. (2007) em Quaraí, por Cassol et al. (2008) em São Borja e por Mazurana et al. (2009), em Santa Rosa.

A erosividade anual de Encruzilhada do Sul variou de 2935,7 MJ mm ha ${ }^{-1} h^{-1}$ em 1964 a 9950,9 MJ mm ha-1 $h^{-1}$ em 1986 (Figura 3). Neste último ano, o potencial erosivo de dois meses (abril e maio) representou $49 \%$ do maior potencial erosivo anual ocorrido no período estudado. Foi de 5534,3 $\mathrm{MJ} \mathrm{mm} \mathrm{ha}^{-1} \mathrm{~h}^{-1} \mathrm{o}$ valor médio obtido para os 31 anos da série de dados pluviográficos (Figura 3), o qual representa o fator "R" da USLE, que pode ser utilizado com segurança para localidades próximas

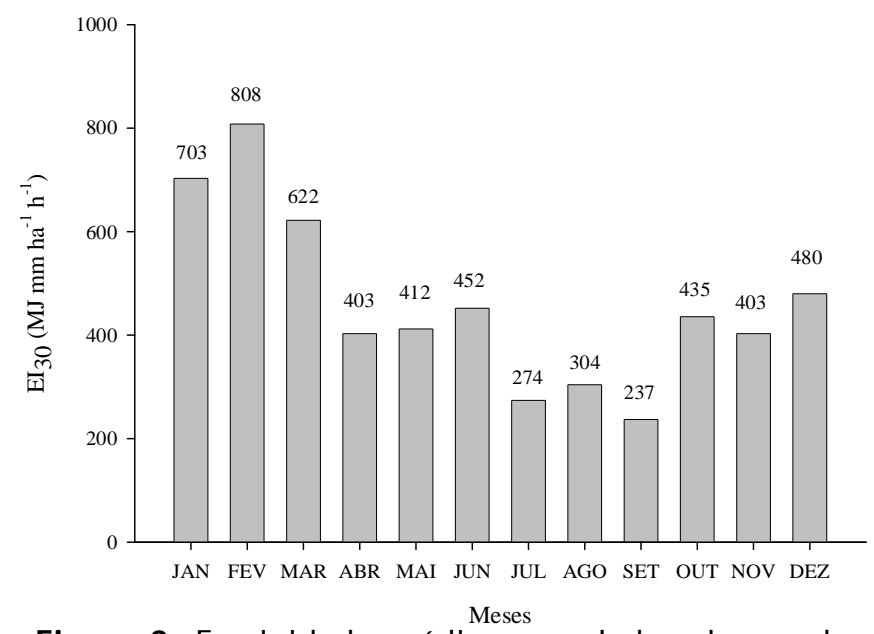

Figura 2. Erosividade média mensal das chuvas de Encruzilhada do Sul, RS, no período de 1958 a 1988, determinada pelo índice $\mathrm{EI}_{30}$

à Encruzilhada do Sul, com características climáticas semelhantes.

Valores de erosividade anual maiores que os determinados para Encruzilhada do Sul foram obtidos para Santa Maria (7.866 MJ mm ha-1 $\mathrm{h}^{-1}$ ) por Cogo et al. (2006), para Quaraí (9.292 MJ $\mathrm{mm} \mathrm{ha} \mathrm{h}^{-1} \mathrm{~h}^{-1}$ ) por Peñalva-Bazzano et al. (2007), para Uruguaiana 


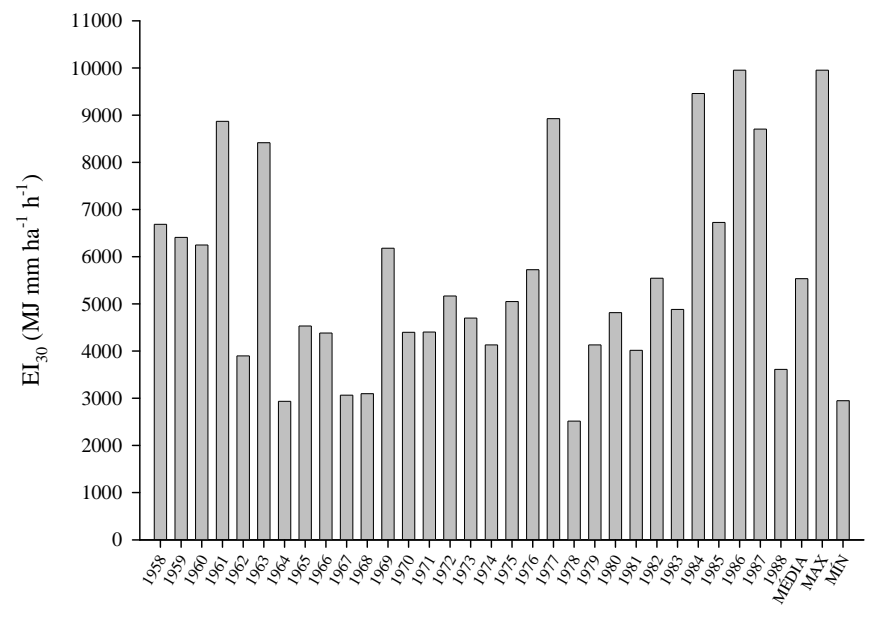

Anos

Figura 3. Erosividade anual das chuvas de Encruzilhada do Sul, RS, determinada pelo índice $\mathrm{El}_{30}$, no período de 1958 a 1988

(8.875 MJ mm ha-1 $\mathrm{h}^{-1}$ ) por Hickmann et al. (2008), para São Borja (9.751 MJ mm ha ${ }^{-1} \mathrm{~h}^{-1}$ ) por Cassol et al. (2008), para Santa Rosa (1.1217 $\mathrm{MJ} \mathrm{mm} \mathrm{ha}^{-1} \mathrm{~h}^{-1}$ ) por Mazurana et al. (2009), todos no Estado do Rio Grande do Sul.

O padrão avançado de chuvas ocorreu em $48 \%$ do número de chuvas erosivas, enquanto dos padrões intermediário e atrasado, cada um teve $26 \%$ (Figura 4). O maior número de ocorrência de chuvas do padrão avançado que se caracteriza pelo pico de maior intensidade no primeiro terço da chuva, do ponto de vista de erosão é, até certo ponto, benéfico, de vez que no início da chuva o solo ainda está mais seco e tem condições de absorver mais água. Em relação à lâmina de chuvas erosivas, a distribuição foi semelhante à distribuição do número de chuvas, com 51, 27 e $22 \%$, respectivamente, para os padrões avançado, intermediário e atrasado. Entretanto, em relação ao potencial erosivo esta distribuição é mais desigual, ocorrendo 55, 27 e 18\% para os padrões avançado, intermediário

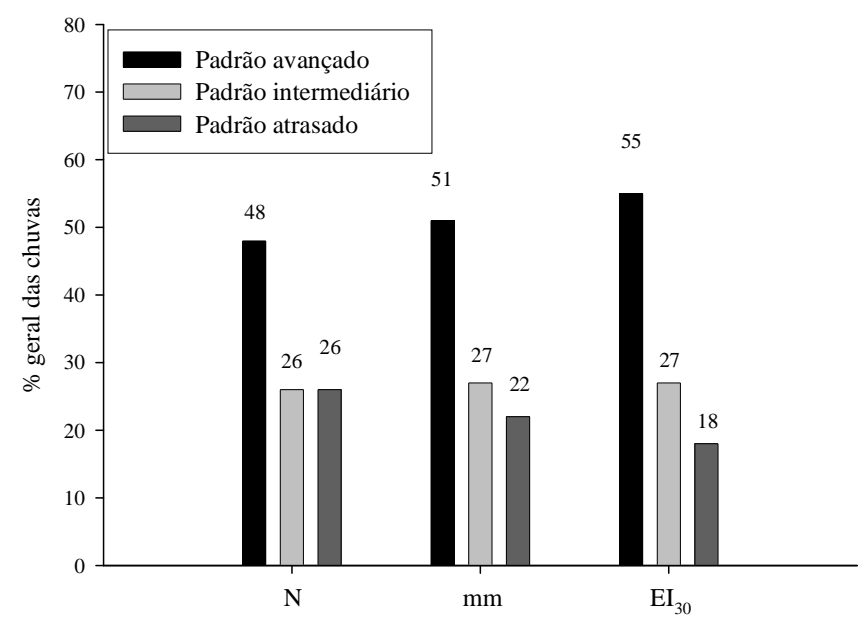

Figura 4. $D$ istribuição da percentagem média anual do número $(\mathrm{N})$, da quantidade média anual $(\mathrm{mm})$ e do índice de erosividade médio anual $\left(\mathrm{EI}_{30}\right.$ ) das chuvas de Encruzilhada do Sul, RS, por padrões hidrológicos, no período de 1958 a 1988 e atrasado. As chuvas de padrão avançado têm potencial erosivo menor já que a resistência do solo é maior no início da chuva, em virtude do menor selamento superficial, desagregação e transporte, do que nas chuvas dos outros padrões (Mehl et al., 2001), o que foi comprovado por Eltz et al. (2001), que verificaram maior erosão em chuvas do padrão atrasado em referência aos padrões intermediário e avançado, já explicado pela alteração da superfície do solo e conteúdo de água no decorrer da chuva.

As relações linear e potencial obtidas por regressão entre o coeficiente de chuvas (Rc) e a erosividade das chuvas (expresso pelo índice $\mathrm{EI}_{30}$ ), não foram significativas (Tabela 3), significando que o coeficiente de chuvas não pode ser utilizado nesta região para estimar o potencial erosivo das chuvas. Esses resultados diferem dos obtidos por vários pesquisadores, com correlações significativas entre o índice de erosividade $\mathrm{EI}_{30}$ e o coeficiente de chuva Rc, entre os quais estão os de Rufino et al. (1993), que obtiveram relação linear para oito regiões do Estado do Paraná, com coeficiente de correlação variando de $r=0,84^{*}$ a $r=0,93^{*}$, de Peñalva-Bazzano et al. (2007) que obtiveram correlação linear significativa $\left(\mathrm{EI}_{30}=-47,35+82,72\right.$ Rc) em Quaraí, RS, e Cassol et al. (2008) que também encontraram correlação linear significativa para São Borja, $\mathrm{RS}\left(\mathrm{EI}_{30}=99,646\right.$ +63,874 Rc). Entretanto, também não se encontraram, em outros estudos, correlações significativas entre o índice $\mathrm{EI}_{30}$ e o coeficiente de chuvas, como foi o caso de Cassol et al. (2007), para Ijuí, RS, de Mazurana et al. (2009) para Santa Rosa, RS, e de Bertol (1994), para Campos Novos, SC.

Tabela 3. Relações da erosividade com as quantidades de chuvas de Encruzilhada do Sul, RS

\begin{tabular}{|c|c|c|c|c|c|}
\hline \multirow[t]{2}{*}{ Mês } & \multirow[t]{2}{*}{$\begin{array}{l}\text { Chuva } \\
\text { média } \\
\text { mensal }\end{array}$} & \multirow{2}{*}{$\begin{array}{l}\text { Coeficiente } \\
\text { de chuva Rc }\end{array}$} & \multirow{2}{*}{$\begin{array}{c}\mathrm{El}_{30} \\
\text { Observado } \\
\text { MJ } \mathrm{mm} \mathrm{ha}^{-1} \mathrm{~h}^{-1}\end{array}$} & \multicolumn{2}{|c|}{$\begin{array}{l}\mathrm{El}_{30} \text { calculado } \\
\mathrm{MJ} \mathrm{mm} \mathrm{ha}^{-1} \mathrm{~h}^{-1}\end{array}$} \\
\hline & & & & Eq. (1) & Eq. (2) \\
\hline Jan & 105,0 & 8,62 & 702,8 & 464,0 & 435,7 \\
\hline Fev & 109,5 & 9,37 & 808,5 & 458,1 & 428,4 \\
\hline Mar & 109,9 & 9,44 & 621,8 & 457,5 & 427,8 \\
\hline Abr & 89,9 & 6,32 & 403,2 & 482,1 & 463,7 \\
\hline Mai & 98,1 & 7,52 & 412,1 & 472,6 & 447,7 \\
\hline J un & 124,4 & 12,10 & 451,9 & 436,6 & 407,0 \\
\hline Jul & 116,0 & 10,52 & 274,0 & 449,0 & 418,6 \\
\hline Ago & 119,5 & 11,16 & 304,2 & 443,9 & 413,6 \\
\hline Set & 108,8 & 9,25 & 237,1 & 459,0 & 429,5 \\
\hline Out & 113,5 & 10,07 & 435,6 & 452,6 & 422,2 \\
\hline Nov & 91,9 & 6,60 & 402,8 & 479,9 & 459,6 \\
\hline Dez & 92,7 & 6,72 & 480,3 & 479,0 & 458,0 \\
\hline Total anual & $1.279,1$ & & $5.534,3$ & $5.534,3$ & $5.211,8$ \\
\hline
\end{tabular}

Eq. (1): $\mathrm{El}_{30}=531,97-7,8855 \mathrm{Rc} ; \mathrm{R}^{2}=0,0074$

Eq. (2): $\mathrm{El}_{30}=671,72 \mathrm{RC}^{-0,201} ; \mathrm{R}^{2}=0,0140$

$R C=p^{2} / P$, donde $p=$ precipitação média mensal $(\mathrm{mm}) \mathrm{e}$

$\mathrm{P}=$ precipitação média anual $(\mathrm{mm})$

O período de retorno e a probabilidade de ocorrência de todos os eventos anuais de erosividade no período 1958 a 1988, são mostrados na Tabela 4. O maior valor de erosividade anual de 9.950,9 $\mathrm{MJ} \mathrm{mm} \mathrm{ha}^{-1} \mathrm{~h}^{-1}$ tem um período de retorno estimado de 32 anos, com probabilidade de ocorrência de 3,1\%; o menor valor de erosividade anual tem período de retorno de um ano e probabilidade de ocorrência de 96,9\%; da mesma 
forma, o valor médio da erosividade anual (Fator R de 5534,3 $\mathrm{MJ} \mathrm{mm} \mathrm{ha-1} \mathrm{h}^{-1}$ para Encruzilhada do Sul,) tem um período de retorno estimado de 2,5 anos, com probabilidade de ocorrência de $40,6 \%$, significando que há probabilidade de, a cada 2,5 anos, ocorrerem chuvas anuais com potencial erosivo igual à média.

Tabela 4. Período de retorno e probabilidade de ocorrência dos valores dos índices anuais de erosividade $\left(\mathrm{El}_{30}\right)$, de Encruzilhada do Sul (RS) durante o período de 1958 a 1988

\begin{tabular}{|c|c|c|c|c|}
\hline Ano & $\begin{array}{c}\text { Índice de } \\
\text { erosividade } \\
\left(\mathrm{El}_{30}\right) \\
\mathrm{MJ} \mathrm{mm} \mathrm{ha}^{-1} \mathrm{~h}^{-1}\end{array}$ & $\begin{array}{l}\text { Número de } \\
\text { ordem } \\
\text { (m) }\end{array}$ & $\begin{array}{l}\text { Período de } \\
\text { retorno } \\
\text { (T) } \\
\text { Ano }\end{array}$ & $\begin{array}{c}\text { Probabilidade } \\
\text { de ocorrência } \\
\text { (Pr) } \\
\%\end{array}$ \\
\hline 1986 & $9.950,9$ & 1 & 32,0 & 3,1 \\
\hline 1984 & $9.457,1$ & 2 & 16,0 & 6,3 \\
\hline 1977 & $8.923,6$ & 3 & 10,7 & 9,4 \\
\hline 1961 & $8.867,9$ & 4 & 8,0 & 12,5 \\
\hline 1987 & $8.705,2$ & 5 & 6,4 & 15,6 \\
\hline 1963 & $8.415,8$ & 6 & 5,3 & 18,8 \\
\hline 1985 & $6.725,1$ & 7 & 4,6 & 21,9 \\
\hline 1958 & $6.684,9$ & 8 & 4,0 & 25,0 \\
\hline 1959 & $6.408,8$ & 9 & 3,6 & 28,1 \\
\hline 1960 & $6.248,9$ & 10 & 3,2 & 31,3 \\
\hline 1969 & $6.180,3$ & 11 & 2,9 & 34,4 \\
\hline 1976 & $5.722,0$ & 12 & 2,7 & 37,5 \\
\hline 1982 & $5.543,0$ & 13 & 2,5 & 40,6 \\
\hline 1972 & $5.168,1$ & 14 & 2,3 & 43,8 \\
\hline 1975 & $5.050,7$ & 15 & 2,1 & 46,9 \\
\hline 1983 & $4.882,8$ & 16 & 2,0 & 50,0 \\
\hline 1980 & $4.814,8$ & 17 & 1,9 & 53,1 \\
\hline 1973 & $4.697,8$ & 18 & 1,8 & 56,3 \\
\hline 1965 & $4.531,6$ & 19 & 1,7 & 59,4 \\
\hline 1971 & $4.402,5$ & 20 & 1,6 & 62,5 \\
\hline 1970 & $4.398,7$ & 21 & 1,5 & 65,6 \\
\hline 1966 & $4.383,3$ & 22 & 1,5 & 68,8 \\
\hline 1974 & $4.129,8$ & 23 & 1,4 & 71,9 \\
\hline 1979 & $4.129,7$ & 24 & 1,3 & 75,0 \\
\hline 1981 & $4.015,1$ & 25 & 1,3 & 78,1 \\
\hline 1962 & $3.898,4$ & 26 & 1,2 & 81,3 \\
\hline 1988 & $3.613,8$ & 27 & 1,2 & 84,4 \\
\hline 1968 & $3.095,7$ & 28 & 1,1 & 87,5 \\
\hline 1967 & $3.065,0$ & 29 & 1,1 & 90,6 \\
\hline 1964 & $2.935,7$ & 30 & 1,1 & 93,8 \\
\hline 1978 & $2.517,3$ & 31 & 1,0 & 96,9 \\
\hline
\end{tabular}

\section{ConclusõEs}

1. O valor do fator "R" da Equação Universal de Perdas de Solos para Encruzilhada do Sul, RS, é de 5.534,3 $\mathrm{MJ} \mathrm{mm} \mathrm{ha}^{-1} \mathrm{~h}^{-1}$, que para os valores do Rio Grande do Sul, pode ser considerado como de médio potencial erosivo.

2. O período de janeiro a março é o mais crítico em relação ao potencial erosivo das chuvas e o período de julho a setembro é o menos crítico.

3. O padrão hidrológico avançado é o de maior ocorrência tanto em número, volume e potencial erosivo das chuvas, seguido dos padrões intermediário e atrasado.

4. Quanto ao valor da erosividade média anual (5.534,3 MJ $\mathrm{mm} \mathrm{ha}^{-1} \mathrm{~h}^{-1}$ ) espera-se que ocorra uma vez a cada 2,3 anos, com probabilidade de ocorrência de $40,6 \%$.

\section{LITERATURA CITADA}

Albuquerque, A. W.; Lombardi Neto, F.; Srinivasan, V. S.; Cataneo, A. Parâmetros erosividade da chuva e da enxurrada correlacionados com as perdas de solo de um solo Bruno Não Cálcico de Sumé-PB. Revista Brasileira de Ciência do Solo, v.22, p.743-749, 1998.

Bertol, I. Avaliação da erosividade da chuva na localidade de Campos Novos (SC) no período de 1981-1990. Pesquisa Agropecuária Brasileira, v.29, p.1453-1458, 1994.

Cassol, E. A.; Eltz, F. L. F.; Martins, D.; Lemos, A. M. de; Lima, V. S. de; Bueno, A. C. Erosividade, padrões hidrológicos, período de retorno e probabilidade de ocorrência das chuvas em São Borja, RS. Revista Brasileira de Ciência do Solo, v.32, p.1239-1251, 2008.

Cassol, E. A.; Martins, D.; Eltz, F. L. F.; Lima, V. S. de; Bueno, A. C. Erosividade e padrões hidrológicos das chuvas de Ijuí (RS), no período de 1963 a 1993. Revista Brasileira de Agrometeorologia, v.15, p.220-231, 2007.

Cogo, C. M.; Eltz, F. L. F.; Cassol, E. A. Erosividade das chuvas em Santa Maria, determinada pelo índice $\mathrm{EI}_{30}$. Revista Brasileira de Agrometeorologia, v.14, p.1-11, 2006.

Edwards, W. M.; Owens, L. B. Large storm effects on total soil erosion. Journal of Soil and Water Conservation, v.46, p.7578, 1991.

Eltz, F. L. F.; Mehl, H. U.; Reichert, J. M. Perdas de solo e água em entressulcos em um Argissolo Vermelho-Amarelo submetido a quatro padrões de chuva. Revista Brasileira de Ciência do Solo, v.25, p.485-493, 2001.

Foster, G. R.; McCool, D. K.; Renard, K. G.; Moldenhauer, W. C. Conversion of the universal soil loss equation to SI units. Journal of Soil and Water Conservation, v.36, p.355-359, 1981.

Hickmann, C.; Eltz, F. L. F.; Cassol, E. A.; Cogo, C. M. Erosividade das chuvas em Uruguaiana, RS, determinada pelo índice $\mathrm{EI}_{30}$, com base no período de 1963 a 1991. Revista Brasileira de Ciência do Solo, v.32, p.825-831, 2008.

Horner, W. W.; Jens, S. W. Surface runoff determination from rainfall without using coefficients. Transactions of the ASCE, v.107, p.1039-1117, 1942.

Lombardi Neto, F. Rainfall erosivity - its distribution and relationship with soil loss at Campinas, Brazil. West Lafayette: Purdue University, 1977. 53p. Dissertação Mestrado

Mazurana, J; Cassol, E. A.; Santos, L. C. dos; Eltz, F. L. F.; Bueno, A. C. Erosividade, padrões hidrológicos e período de retorno das chuvas de Santa Rosa, RS. Revista Brasileira de Engenharia Agrícola e Ambiental, v.13, p.975-983, 2009.

Mehl, H. U.; Eltz, F. L. F.; Reichert, J. M.; Didoné, I. A. Caracterização dos padrões de chuva ocorrentes em Santa Maria (RS). Revista Brasileira de Ciência do Solo, v.25, p.475483, 2001.

Morais, L. F. B de; Mutti, L. S. M.; Eltz, F. L. F. Índices de erosividade correlacionados com perdas de solo no Rio Grande do Sul. Revista Brasileira de Ciência do Solo, v.25, p.485-493, 1988. 
Peñalva-Bazzano, M. G.; Eltz, F. L. F.; Cassol, E. A. Erosividade, coeficiente de chuva, padrões e período de retorno das chuvas de Quaraí, RS. Revista Brasileira de Ciência do Solo, v.31,p.1205-1217, 2007.

Rufino, R.; Biscaia, R. C. M.; Merten, G. H. Avaliação do potencial erosivo da chuva do estado do Paraná, através de pluviometria: Terceira aproximação. Revista Brasileira de Ciência do Solo, v.17, p.439-444, 1993.
Schwab, G. O.; Frevert, R. K.; Edminster, T. W.; Barnes, K. K. Soil and Water Conservation Engineering. 3. ed. New York: John Wiley \& Sons, 1981. 525p.

Wischmeier, W. H. A rainfall erosion index for a universal soilloss equation. Soil Science Society of America. v.23, p.246$249,1959$.

Wischmeier, W. H.; Smith, D. D. Predicting rainfall erosion losses - a guide to conservation planning. Washington: USDA. 1978. 58p. Agriculture Handbook 537 